\title{
Archéopages
}

Archéopages

Archéologie et société

Hors-série 3 | 2012

Nouveaux champs de la recherche archéologique

\section{Le monde rural au second âge du Fer : des avancées considérables, des perspectives illimitées}

Geertrui Blancquaert, Thierry Lorho et François Malrain

\section{(2) OpenEdition}

Édition électronique

URL : https://journals.openedition.org/archeopages/706

DOI : 10.4000/archeopages.706

ISSN : 2269-9872

\section{Éditeur}

INRAP - Institut national de recherches archéologiques préventives

\section{Édition imprimée}

Date de publication : 1 janvier 2012

Pagination : 147-153

ISSN : $1622-8545$

\section{Référence électronique}

Geertrui Blancquaert, Thierry Lorho et François Malrain, « Le monde rural au second âge du Fer : des avancées considérables, des perspectives illimitées », Archéopages [En ligne], Hors-série 3 | 2012, mis en ligne le 01 janvier 2012, consulté le 26 février 2023. URL : http://journals.openedition.org/ archeopages/706 ; DOI : https://doi.org/10.4000/archeopages.706 


\section{Le monde rural au second âge du Fer: des avancées considérables, des perspectives illimitées}

\author{
Geertrui Blancquaert \\ Inrap, DRAC-SRA Champagne-Ardenne \\ Thierry Lorho \\ DRAC-SRA Bretagne, UMR 6566 «Civilisation Atlantiques et \\ archéo-sciences » \\ François Malrain \\ Inrap, UMR 7041 «Archéologies et Sciences de l'Antiquité »
}

D ix ans déjà que l'Inrap a été fondé et, pour commémorer cet événement, nous allons exposer les apports de l'archéologie préventive à la connaissance du monde rural au second âge du Fer. L'interprétation du terme «aedificia» de la Guerre des Gaules et la traduction de «native farm» par «ferme indigène» (terme que certains emploient toujours !) appartiennent à l'histoire de la recherche antérieure à l'archéologie préventive. Aux kilomètres parcourus dans le ciel par les avions en quête de clichés sur des habitats enfouis ont succédé les kilomètres de tranchées qui ont permis de faire surgir une multitude de sites insoupçonnés. Si chacune de ces pratiques participent à nourrir la recherche, l'interprétation des textes antiques ou le décryptage des vastes idéogrammes inscrits sur le sol ont trouvé leurs limites, tandis que l'analyse des sites fouillés apparaît infinie. En une trentaine d'année nous sommes passés de l'interprétation d'un mot, «aedificia», à des hypothèses sur l'organisation socio-économique et l'appréciation d'une planification raisonnée du territoire, ainsi que l'étude des entités culturelles des peuples qui occupaient globalement la France d'aujourd'hui. Comment ce parcours s'est-il construit et vers quoi nous conduit-il ? Ces deux questionnements guideront notre propos.

Chronique d'un parcours : de la truelle à la pelle mécanique. La documentation partielle et lacunaire s'enrichit, entre les années 1980 et 1990, des opérations de terrain. Timidement apparaissent alors les premiers articles sur les établissements ruraux comme celui de Conchil-leTemple «la Frénésie » dans le Pas-de-Calais (Leman-Delerive, Piningre, 1981) et de Héret/ Beligneux dans l'Ain (Vicherd et al., 1983). Au début de la décennie suivante, le corpus s'étoffe. Les opérations archéologiques dans les bassins carriers (Aisne, Bassée, Oise) donnent accès, grâce à de vastes décapages, à l'intégralité des habitats ruraux, mais également à leur environnement immédiat. Une réflexion méthodologique s'engage alors sur les techniques à mettre en place pour en extraire le maximum d'informations.

La référence en la matière est fournie par la fouille des sites paléolithiques qui enregistrent finement chacun des mobiliers dans l'optique de projections spatiales. En simultané, la discipline voit l'apparition des systèmes d'informations géographiques, encore limités à l'intra-site, et intègre cette démarche dans sa pratique. La fouille du site de Chevrières (Oise) fut conduite selon ce protocole : pendant plus d'une année, une douzaine de personnes a fouillé à la truelle les 400 mètres linéaires de fossés de l'enclos délimitant l'exploitation agricole. Fouille en quinconce, coupes longitudinales et transversales ont permis de collecter le mobilier par mètre linaire et par couche stratigraphique. L'étude a démontré l'efficacité de la méthode : les cartes de répartition de mobiliers donnent accès à une lecture que l'on peut qualifier d'ethnographie des habitats ; elles aident à interpréter la fonction des bâtiments, des aires de travail et autorisent des hypothèses sur les statuts sociaux des occupants. En 1994, la parution du colloque Les installations agricoles de l'âge $d u$ Fer en Île-de-France (Buchsenschutz, Méniel, 1994), puis en 2000 Les installations agricoles de l'âge du Fer en France septentrionale (Marion, Blancquaert, 200o) proposaient, en sus des présentations de sites et des premières synthèses, une mise au point méthodologique. Par exemple, la fouille complète des tronçons de fossés au détriment d'autres, ou encore des sondages irrégulièrement répartis y étaient jugés inadaptés. Au fur et à mesure des opérations et des discussions qui ont suivi, la méthode d'intervention a été affinée. L'ensemble des protohistoriens reconnaît désormais que seule la fouille intégrale des fossés est efficace. Elle doit suivre un protocole rigoureux dans lequel la collecte du mobilier doit s'appliquer à tout ce qui est d'origine anthropique et doit prendre en compte des prélèvements en vue de l'analyse de l'environnement dans lequel le site se développe (Malrain, 2000). Cette prise en considération de l'ensemble des données, qui seule peut rendre compte de l'organisation d'une société, nécessite un investissement important sur le terrain.

Mais les premières expériences manuelles qui s'ensuivirent, sont apparues bien vite trop chronophages pour pouvoir être étendues à l'ensemble des opérations. La fouille manuelle et exhaustive apparaissait, en effet, inadéquate dans les délais impartis à l'archéologie préventive, ce qui est toujours d'actualité. De nouvelles pratiques se sont donc peu à peu imposées. Elles ne sont, en aucun cas, le fruit du hasard, mais le résultat d'une longue maturation scientifique. La pelle mécanique a été jugée l’outil le plus idoine pour concilier temps, moyens et résultats, même si des interventions manuelles seront toujours nécessaires dans les secteurs les plus critiques. Mais les procédés mécaniques permettent d'avoir accès à toute la documentation matérielle d'un site dans la mesure où ils rendent possible la systématisation de la fouille intégrale. La comparaison entre les gisements fouillés manuellement ou mécaniquement a montré que la mécanisation n'a en rien modifié les résultats que l'on en est en droit d'attendre à l'issue d'une fouille car, léventuelle déperdition de mobilier peut être compensée par des tamisages comme cela a été expérimenté dans l'Aube (Riquier, Grisard, 2011). La pelle mécanique n'a donc pas constitué un frein à la recherche, bien au contraire : dans certains cas, son usage a révélé des indices qu'une fouille manuelle aurait peiné à mettre en évidence. Elle a 


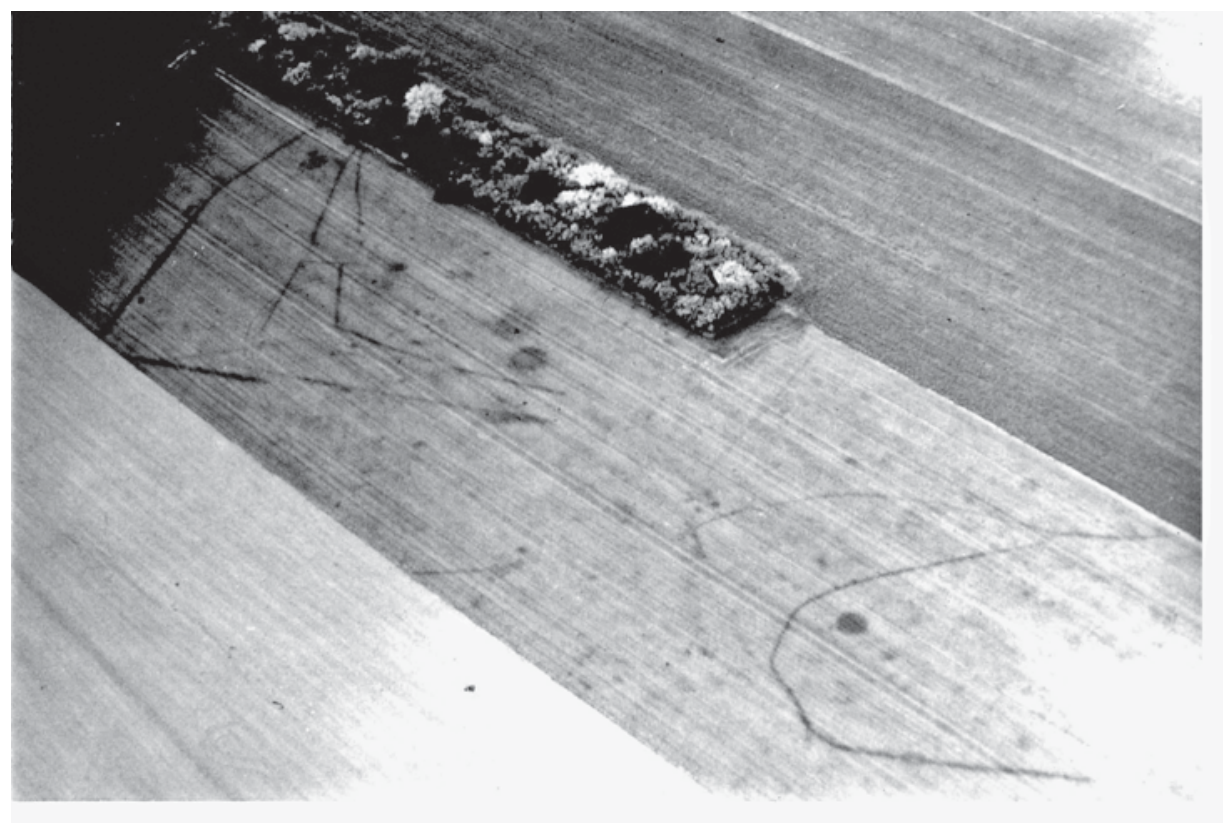

$\stackrel{\infty}{g}$

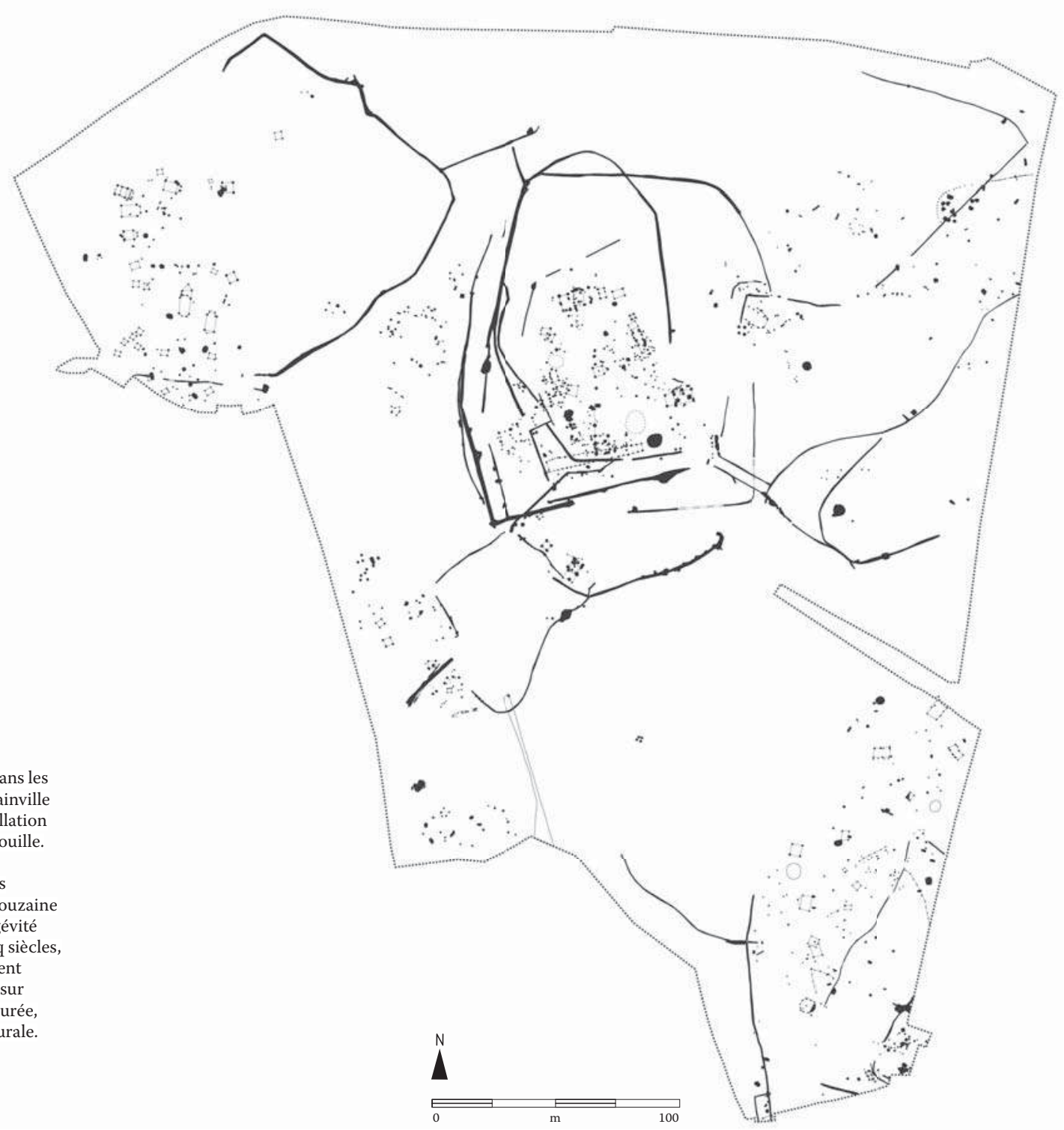

ig. 1] Photographie aérienne dans les années 1970 du site de Poulainville (Somme), classé sous l'appellation «ferme indigène » avant la fouille.

[Fig.2] Le même site relevé après

décapage extensif sur une douzain

d'hectares, en 2005. La longévité

de l'occupation, près de cinq siècles,

et les tombes associées, livrent

des renseignements inédits sur

l'évolution, dans la longue durée

d'une petite communauté rurale. 
ainsi donné la possibilité, quand son équipement le permet (godet large), de procéder à un curage planimétrique des fossés, permettant de déceler des aménagements particuliers comme les traces organiques liées à la décomposition du bois qui, parfois, en tapisse les parois. Les aménagements réalisés au fond des fossés et qui semblent être liés à la gestion de l'eau, peuvent aussi être mis en évidence plus aisément. Ces installations, encore peu reconnues, ont des répercussions importantes sur notre compréhension de l'organisation initiale du site. Elles impliquent un recours aux plans préconçus et une pression accrue sur l'environnement par l'usage plus intensif du bois.

L'un des développements récents de la recherche s'oriente vers des dépôts particuliers qui ponctuent les remplissages des fossés. Si les amas de détritus localisés non loin des maisons sont facilement localisables, il n'en est pas de même pour ces objets ou ossements humains déposés volontairement et dont la nature explique qu'ils ne se trouvent pas mêlés aux rejets de la vie quotidienne (Barral et al., 2007). Là encore, sans le secours de la pelle mécanique, il faudrait brasser de grands volumes de terre manuellement pour avoir une chance de les découvrir.

Jusqu'aux années 2000 , le socle des pratiques d'interventions est ainsi posé, mais celles-ci évoluent toujours et nombre de perfectionnements et d'ajustements sont encore nécessaires.

Un peu de recul s'impose... De manière générale, l'archéologie préventive a permis d'instaurer, en amont de la fouille, la systématisation des sondages en tranchées. Elle a également suscité le raisonnement sur de vastes superficies et favorisé l'exploitation statistique des données collectées massivement. L'action préventive, tranśposée dans une loi, planifiée préalablement aux aménagements territoriaux, a permis d'intervenir dans des zones jusque-là non explorées, non renseignées et donc souvent jugées sans intérêt.

Si cette discipline est conditionnée par l'aménagement territorial, en opposition à l'archéologie programmée, quant à elle libre de ses choix, il n'en demeure pas moins que cet assujettissement peut être considéré comme émancipateur. On s'étonne donc, en 2011, de lire encore sur certains panneaux d'exposition d'organisation interinstitutionnelle que « [...], c'est le hasard qui permet la découverte de ces sites (de lâge du Fer), par exemple lors de la construction de routes et de voies ferrées, parking $[\ldots]$ ».

Les opérations préventives, sondages et fouilles réunis, ont rapidement démontré que l'image fournie par la prospection aérienne était bien en deçà de la réalité [Fig.1-2]. Seules les opérations de terrain permettent véritablement d'approfondir l'histoire de la société gauloise, ce que les indices par exemple collectés par des techniques de prospection non intrusives n'autorisent aucunement.

Ceci étant précisé, il convient également de porter un regard critique sur la manière de procéder lors des diagnostics. Les sondages systématiques à $10 \%$ doivent être considérés comme un minimum, un filtre qui s'avère insuffisant au repérage et à la compréhension de certains types de gisements, par exemple des unités d'habitat ou funéraire de superficie restreinte, mais pour autant essentielles à l'appréciation des sociétés anciennes. Il nous faut donc nuancer le succès d'une technique devenue la norme et garder à l'esprit le rôle majeur des projets qui ont bénéficié d'un décapage intégral au moment du diagnostic, suivi par une fouille exhaustive, tels que les programmes archéologiques développés dans les grandes vallées du bassin parisien. Ainsi, c'est grâce au suivi archéologique systématique sur des centaines hectares entièrement découverts que les occupations les plus modestes sont mises en évidence. La table ronde de Châlonsen-Champagne, qui a traité de la représentativité des sites par rapports aux surfaces ouvertes, rend bien compte des enjeux scientifiques conditionnés par la taille des décapages (Brun et al., 2006). Pour toutes les périodes, ces petits sites peu spectaculaires prennent leur importance lorsque l'objectif est de comprendre l'organisation des sociétés dans toute leur diversité. Actuellement,et selon les régions, ils ne sont plus que rarement traités car ils sont considérés comme sans intérêt ou anodins. Détailler davantage le sujet ici n'a pas de sens car, il touche toutes les périodes et relève d'une politique générale de la recherche (Brun et al. 2006).

Or, les investigations exhaustives menées sur des micro-aires géographiques, conjuguées à la mise en place de bases de données homogénéisées (archéologiques, archéozoologiques, carpologiques, palynologiques etc.) ont offert (sans préjugés) des éléments fondamentaux à la compréhension de la société gauloise. Les données archéologiques collectées et étudiées sur ces opérations de surface importantes peuvent être considérées comme autant d'échantillons représentatifs pour l'étude des sociétés du passé. Les acquis scientifiques qu'elles ont livrés ont permis l'interprétation des gisements partiellement découverts lors des projets linéaires qui représentent une bonne part de l'activité de l'Inrap (Malrain, Blancquaert, 2011 ; Blancquaert et al., 2011 ; Blancquaert, Depaepe, Sous presse). Pour parfaire l'étude de la ruralité laténienne, il serait bon qu'une réflexion soit engagée sur l'opportunité d'investigations approfondies sur des zones tests...

De l'établissement rural aux entités culturelles. L'évolution des procédés d'interventions sur le terrain et des protocoles de fouilles ont rapidement engendré une documentation prolixe. Face à l'afflux de cette importante masse de données, les protohistoriens ont fait preuve d'une belle réactivité. La manifestation scientifique de 1994 sur les installations agricoles de lâge du Fer en Île-de-France s'est révélée fondamentale car elle est le point d'orgue d'une double création, celle d'un inventaire indispensable à la gestion et l'étude de la documentation, d'une part, et celle d'un programme collectif de recherche, incontournable pour promouvoir ce recensement d'autre part (Buchsenschutz, Méniel, 1994).

Les années qui suivent voient l'apparition de monographies, dont celles de la ferme «le 
Boisanne » à Plouer-sur-Rance en Côtes-d'Armor (Menez, 1996) et « le Camp du Roi » à Jaux dans l'Oise (Malrain et al., 1996). C'est aussi l'époque où l'intérêt porté aux campagnes dépassera la seule période protohistorique et trouvera une expression dans le colloque De la ferme indigène à la villa gallo-romaine, organisé et publié par l'association AGER (Association d'étude du monde rural galloromain) à Amiens (Bayard, Collart, 1996).

Au moment de la parution des actes du colloque Les installations agricoles de l'âge du Fer en France Septentrionale en 200o, l'inventaire entrepris en 1995 comprend près de 300 sites. Il a permis de livrer les premières synthèses portant sur la typochronologie des enclos, aussi bien que sur des thématiques socio-économiques, qu'il s'agisse de l'exploitation du sel, de stockage alimentaire, de l'évolution des productions agricoles ou encore de pratiques funéraires en contexte d'habitat (op. cit., 200o). La même année, ou peu de temps après, les travaux universitaires engagés sur ce domaine de recherche sont soutenus (Malrain, 2000 ; Gransar, 2001 ; Matterne, 2002). Ils forment, avec les études de faune développées par Patrice Méniel et Ginette Auxiette, l'ossature de l'ouvrage Les paysans gaulois. Cette synthèse, outre l'étude des sites en eux-mêmes, intègre ceux-ci dans leur environnement (Malrain et al., 2002).

Le paysage et les productions des sites constituent des points forts de la recherche sur cette période. L'influence des prospections a conditionné l'interprétation des sites : un enclos, quelques structures et bâtiments, le qualificatif de «ferme» implicitement utilisé pour désigner l'établissement, sans qu'aucun indice ne soit réuni pour argumenter cette dénomination. Interviennent, lors des opérations de terrains avec des moyens financiers un peu plus soutenus, des prélèvements sédimentaires dans le but d'analyses paléoenvironnementales. Ces analyses et la ténacité des chercheurs ont conduits aux premiers résultats probants. Les établissements ruraux livraient enfin les premiers renseignements sur leurs productions. À leurs abords, champs et pâtures se substituent à la forêt. Le blé amidonnier, l'épeautre et l'orge vêtue sont des espèces abondamment cultivées dans le nord de la Gaule. En perfectionnant peu à peu les techniques de prélèvements et de tamisages, les savoirs gagnent du terrain. Modes d'ensemencements, entretien des espaces cultivés, traitements des céréales de la moisson à la consommation, constituent des éléments de vastes chaînes opératoires peu à peu documentées. Les études sur le long terme rendent perceptibles l'évolution sur les denrées produites. Si aux $\mathrm{V}^{\mathrm{e}}$ et $\mathrm{IV}^{\mathrm{e}}$ siècles, plusieurs espèces sont cultivées ensemble de manière à garantir au moins une récolte, peu à peu cette pratique fut abandonnée pour ensemencer seulement une espèce par champs. Aussi, l'apport de la carpologie va bien au-delà d'une simple science botanique : elle intègre le discours archéologique et devient l'une des clés de la détermination fonctionnelle des sites, de leur environnement, ainsi que des changements d'organisation des sociétés durant les cinq siècles qui précédent notre ère (Zech-Matterne et al., 2009). En concomitance, la palynologie esquisse le paysage des sites, la lecture des diagrammes laisse entrevoir une ouverture importante du milieu naturel à cette période (Leroyer et al., 2009). Quant aux études de faunes, si elles avaient déjà montré, depuis quelques décennies, toute leur utilité pour l'analyse socio-économique des sites, elles fournissent alors nombre d'arguments sur le rang hiérarchique des habitats et les statuts sociaux de leurs habitants. En regard croisés, l'archéologie rurale de l'âge du Fer et les sciences qui s'y associent permettent de montrer qu'il existait différents niveaux d'exploitation agricole gradués en fonction des vestiges immobiliers, mobiliers et des aliments consommés.

Au gré des aménagements territoriaux, les cartes de répartition des habitats ruraux se densifient, offrant à chaque région la possibilité d'apporter sa touche à l'écriture de l'histoire rurale laténienne. Approches et études se diversifient, explorent des axes variés : ici, on mettra l'accent sur le terroir lié aux fermes, comme en Basse-Normandie, à l'exemple d'Ifs (Calvados), où sept établissements et leur parcellaire, étudiés sur 50 hectares, révèlent une densité particulièrement forte. Une étude sur un secteur élargi atteste d'un maillage agraire d'orientation similaire qui unit de nombreuses «fermes» entre elles sur une zone de $50 \mathrm{~km}^{2}$ (Le Goff, 2009). Là, on s'interrogera sur la notion de ferme, de hameau, de village ou encore sur l'architecture des bâtiments (Maguer, 2005). Dans d'autres secteurs géographiques, on cherchera à mettre en valeur la spécificité régionale des sites au regard des autres découvertes en Gaule, à l'instar du dossier sur les sites de l'est qui démontre que, en Lorraine, Alsace et Champagne-Ardenne, la structuration de l'espace agraire est très différente, dans la mesure où la configuration ouverte de l'habitat et l'absence de parcellisation perdurent au moins jusqu'au milieu du $\mathrm{II}^{\mathrm{e}}$ siècle (Fichtl, 2005). Dans le Sud de la France, alors que l'on s'interrogeait encore au milieu des années 1990 sur l'existence des «fermes indigènes » dans cet espace géographique (Sauvage, 1996), on sait désormais qu'elles y sont bel et bien présentes et, bien mieux encore, les travaux récents autour de Nîmes ont montré une forte occupation de la plaine : habitats, parcellaires et nécropoles s'y inscrivent dans un paysage structuré par les cours d'eau (Séjalon et al., 2009 ; Breuil, 2010). Le mobilier découvert lors des fouilles fait aussi l'objet d'une analyse spécifique. L'instrumentum, qui permet de mieux cerner les activités qui y étaient pratiquées, nécessite une connaissance approfondie des outils et de leurs usages. Trop souvent négligés car considérés comme peu prestigieux au regard des armes et des parures, leur analyse apporte pourtant bien des éclairages tant sur les pratiques de culture et d'élevage que sur les techniques de construction, la fabrication d'objets, etc. (Nillesse, 2009). Bien d'autres exemples d'avancées scientifiques pourraient encore être évoqués et on nous pardonnera de ne pouvoir les citer tous. 
Les inventaires : outil essentiel à la recherche.

Près de 10 années après le premier inventaire, la nécessité de disposer d'une base de données renouvelée s'est à nouveau fait sentir lorsque le choix de l'un des colloques de l'Association Française pour l'Étude de l'Âge du Fer a porté sur les habitats et paysages ruraux en Gaule et sur d'autres régions du monde celtique (Bertrand et al., 2009). Dans ce cadre, les présentations de sites, qui ne peuvent être que monographiques, n'étaient pas appropriées, seuls des exposés de synthèse thématiques devaient être privilégiés. Mais comment réaliser des synthèses à partir de données dispersées et hétérogènes ? Très rapidement il a été établi que le moyen le plus efficace était de réactiver l'inventaire des sites de 1995, en l'étendant à l'ensemble de la France. Il s'impose comme l'outil coordonnateur de la production scientifique. Les méthodes, les questionnements, les savoirs ayant évolué en une décennie, il fut convenu de revoir totalement la conception de l'enregistrement codifié des sites en le rendant plus performant grâce aux nouvelles technologies dont l'amélioration principale consiste à lier les données et le système d'information géographique (Blancquaert et al., 2009). La revue Archéopages s'est fait l'écho à la fois de la mise en place de cette base de données, de son organisation, des résultats qu'elle a produits et de son potentiel; il n'est donc pas utile de revenir ici sur cette étape de la recherche. C'est d'ailleurs à partir de l'un des articles portant sur les rythmes de créations et d'abandons des établissements (Nouvel et al., 2009), que fut par la suite décidé d'étendre cette réflexion à l'ensemble de la France septentrionale. L'ouvrage, en cours d'achèvement, livre des résultats novateurs car, pour la première fois à partir de données traitées uniformément, les résultats de chacune des régions permettent de mettre en évidence les spécificités propres à chacune d'elles. Aux habitats fortement structurés et de longue durée à l'ouest, s'opposent des habitats aux rythmes courts et très largement ouverts à l'est, tandis que les sites localisés entre ces deux pôles présentent une situation intermédiaire. Mais ce qui apparait le plus remarquable, ce sont les possibilités offertes par ce corpus qui permet, par une simple projection cartographique des établissements au cours du temps, de comparer les grandes tendances avec des faits historiquement connus comme celui de l'épisode de la Guerre des Gaules qui se marque par une baisse brutale du nombre d'occupations, tout comme se mesure un « exode rural » au moment où se mettent en place les grandes agglomérations et les villes. Les fluctuations, lors des cinq siècles qui précédent notre ère, peuvent aussi être mises en corrélation avec les données environnementales. Aux pics les plus importants d'établissements correspondent aussi des déboisements importants, tandis que les cultures tendent à une plus forte spécialisation régionale. De plus, il a été possible, grâce aux études récentes en matière de climat, d'apercevoir l'impact éventuel (sans parler de déterminisme) des variations climatiques sur les agissements de l'homme (Büntgen et al., 2011 ; Blancquaert et al., à paraître).

L'échelle d'analyse, dont on peut espérer qu'elle couvrira bientôt l'ensemble de la France, croisée avec les données des sciences paléoenvironnementales, multiplie les axes d'études et ouvre à des horizons infinis.

... et pour l'avenir. On a pu le constater, la recherche sur les campagnes gauloises, comme toute recherche, touche des problématiques longues à traiter. Il a fallu plus de 30 ans pour en arriver là. Car, en parallèle à l'évolution des méthodes et techniques de détection et de fouille indispensables à l'acquisition des données, il a fallu celle des mentalités acceptant une approche plus globale de l'archéologie et encourageant une plus forte interdisciplinarité. Il était en effet nécessaire de dépasser le site en soi pour pouvoir étudier, entre autres, les pratiques culturales, les réseaux (voiries, densité des occupations...) que tissent les établissements ruraux au fil du temps, le paysage sans cesse modelé par l'homme... afin de transmettre ce patrimoine. Il convient donc non pas de fouiller « une ferme», mais plusieurs, voire beaucoup, et cela sur des superficies les plus vastes possibles. On revient ici sur un point évoqué précédemment : comment bénéficier d'occasions futures pour explorer des zones tests dans l'objectif de compléter et d'affiner nos connaissances sur le monde rural au second âge du Fer ? Ce sujet intéresse avant tout les chercheurs chargés de l'instruction des dossiers d'aménagement du territoire et des prescriptions car ils en sont pleinement tributaires. Face à l'évidence qu'il est impossible de tout fouiller, il faudra à l'avenir être en mesure de pouvoir développer des secteurs où l'on conjugue les objectifs de la recherche et des travaux publics, même s'il s'agit, du moins dans un premier temps, de superficies segmentées.

Quant aux acquis scientifiques esquissés, il est manifeste que ces avancées, faites dans le monde rural des cinq siècles précédents notre ère, doivent, à terme, intégrer les périodes précédent (phénomène des principautés Hallstattiennes) et suivant (romanisation) la séquence étudiée, dans la perspective de mieux appréhender les raisons et l'intensité des changements. Et, toujours dans cette même optique globalisante, le travail futur doit s'ouvrir davantage sur l'interaction des différents types d'occupations, qu'il s'agisse des établissements ruraux et des agglomérations, fortifiées ou non, des sanctuaires ou nécropoles... pour saisir la société gauloise dans toutes ses dimensions.

L'immense corpus des établissements ruraux fouillés dans le cadre de l'archéologie préventive et, plus particulièrement ceux ceints par un système fossoyé, constitue également un référentiel typochronologique fondé sur la morphologie des enclos, leur superficie... permettant de revisiter le non moins imposant corpus d'établissements détectés en prospection aérienne enregistrés sur la carte archéologique nationale. Ensemble, ils offrent une image plus «fournie» de la répartition 
des sites de lâge du Fer qu'il conviendra d'exploiter à sa juste valeur.

Enfin, ajoutons une dernière réflexion prospective qui consiste à encourager la recherche intéressant la caractérisation fonctionnelle optimale, autrement dit la diversité des activités pouvant avoir lieu sur ces établissements. Pouvoir mettre en valeur leurs spécialisations sous-entend ouvrir davantage d'alternatives favorables à l'enrichissement de nos connaissances du tissu rural, cette indispensable composante d'une société d'où naîtront les premiers États. Le présent exemple illustre parfaitement que l'archéologie préventive, dans sa capacité d'adaptation au terrain, dans sa participation active à la mise au point de méthodologies de fouilles nouvelles et maintenant dans sa compétence à gérer des sommes d'informations conséquentes, répond pleinement à sa mission de recherche.

Barral P., Daubignez A., Dunning C., Kaenel G., RouliereLAMBERT M.-J., 2007 : Lâge du Fer dans l'arc jurassien et ses marges. Dépôts, lieux sacrés et territorialité à l'âge du Fer, Actes du XXIXe colloque international de l'AFEAF, Bienne, 5-8 mai 2005, vol. 2, Presses universitaires de Franche-Comté, $891 \mathrm{p}$.

BAYARD D., CollarT J.-L., (DIR.), 1996 : De la ferme indigène à la villa romaine : la romanisation des campagnes de la Gaule, Actes du II colloque de l'association AGER, Amiens 23-25 septembre 1993 Revue Archéologique de Picardie, nº́sécial, 11, 336 p.

Blancquaert G., Lorho T., Malrain F., Menez Y., 2009 : «Bilan et perspectives de recherche sur les sites ruraux au second l'âge du Fer», in Bertrand I., Duval A., Gomez de Soto J., Maguer P. (Dir.), p. 5-23.

Blancquaert G., Malrain F., StÄuble H., Vanmoerkerke J. (ÉD.), 2011 : Understanding the past: a matter of surface area, Actes de la XIII Session de l'Association Européenne des Archéologues, Zadar, Croatie, 2007, British Archaeological Report (International Series), $\mathrm{n}^{\circ} 2194$, p. 162.

Blancquaert G., Leroyer C., Lorho T., Malrain F., ZeChMATTERNE V., à paraître : « Rythmes de créations et d'abandons des établissements ruraux du second âge du Fer et interactions environnementales ", in Bertoncello F., Berger J.-F., CARozza L., Braemer F., Depaepe P. (DIR.), Variations environnementales, mutations sociales : natures, intensités, échelles et temporalités des changements, Actes des XXXII Rencontres internationales d'archéologie et d'histoire d'Antibes.

Blancquaert G., Depaepe P., Sous presse : « Des sondages systématiques et des fouilles réalisées sur des grandes superficies. Deux techniques au service d'un passé enseveli », in EAC Occasional Paper, ${ }^{\circ}$ 6, p. 47-56.

Bertrand I., Duval A., Gomez De Soto J., Maguer P. (Dir.), 2009: Habitats et paysages ruraux en Gaule et regards sur d'autres régions du monde celtique, Actes du XXXI colloque international de l'AFEAF, Chauvigny, 17-20mai 2007, Mémoire XXXV-2009, Chauvigny, éd. Association des publications Chauvinoises, $541 \mathrm{p}$.

BREUIL J.-Y., 2010 : « Nîmes : de la ville gauloise à la Nemausus romaine », in Ouzoulias P., Tranoy L. (DIR.), Comment les Gaules devinrent romaines, Paris, La découverte, p. 125-143.

BRun P., MARCigny C., VANMOERKERKe J. (DIR.), 2006 : Une archéologie des réseaux locaux. Quelles surfaces étudier pour quelle représentativité ?, Actes de la table ronde de Châlons-en-Champagne, 14-15 juin 2005, Les nouvelles de l'archéologie, n¹04-105.

Buchsenschutz O., MÉNIEL P. (DIR.) 1994 : Les installations agricoles de lâge du Fer en Ile de France, Paris, ENS (Études d'histoire et d'archéologie, IV), 299 p.

Büntgen U., Tegel W., Nicolussi K., Mccormick M., Frank D., Trouet V., Kaplan J.O., Heussner K.-U., Wanner H., LUterbacher J., EsPer J., 2011 : « Years of European Climate Variability and Human Susceptibility ", Science, 331, p. 578-58o.

FICHTL S. (DIR.), 2005: Hiérarchie de l'habitat rural dans le Nord-Est de la Gaule à La Tène moyenne et finale, Archaeologia Mosellana, n 6, 439 p.

GrANSAR F., 2001 : Le stockage alimentaire à l'âge du Fer en Europe nord-occidentale, thèse de doctorat (en cours), Université de Paris I Panthéon-Sorbonne.

LE GOFF E., 2009 : « Habitats, terroir et paysage rural : Aménagement et structuration du territoire et de la campagne gauloise, Ifs Z.A.C. « Object'Ifs Sud » », in Bertrand I., Duval A., Gomez de Soto J., Maguer P. (DIR.), p. 93-107.

Leman-Delerive G., Piningre J.-F., 1981 : « Les structures d'habitat du deuxième âge du Fer de Conchil-le-Temple (Pas-de-Calais), premiers résultats ", in L'âge du Fer en France septentrionale, Actes du IIIe colloque de l'AFEAF, Châlons-sur-Marne, 1979, Mémoire de la Société Archéologique Champenoise, $\mathrm{n}^{\circ}$ 2, Suppl. n 1, Reims, p. 319-330.
Leroyer Ch., Boulen M., Marguerie D., Lorho T., Prat B., Argant J., Allenet De Ribemont G. (contrib.), Aoustin D. (Contrib.), Diot M.-F. (CONTrib.), Gaudin L. (CONTrib.), Guenet P. (Contrib.), Latour-Argant C. (CONTrib.), Marembat L. (Contrib.), Perrière J., Tixier C. (Contrib.), Vivent D. (CONTRIB.), 2009: « Base de données et SIG palynologiques sur l'âge du Fer en France : une autre approche du paysage végétal et de son anthropisation », in Bertrand I., Duval A., Gomez de Soto J., MAGUER P. (DIR.), p. 447-468.

MAGUER P., 2005: « L'architecture des bâtiments de La Tène dans le sud du Maine-et-Loire et en Vendée, étude de cas », in BuCHSENSCHUTZ O. (DIR.), Architectures protohistoriques en Europe occidentale du Néolithique final à l'âge du Fer, Paris, CTHS, p. 331-345.

Malrain F., Gransar F., Matterne V., Le Goff I., 1996 : « Une ferme de La Tène D1 et sa nécropole : Jaux « le Camp du Roi » (Oise) », Revue Archéologique de Picardie, nº 3-4, p. 245-306.

Malrain F., 2000 : Fonctionnement et hiérarchies des fermes dans la société gauloise du III siècle à la période romaine : l'apport des sites de la moyenne vallée de l'Oise, thèse de doctorat, Université de Paris I Panthéon-Sorbonne, $436 \mathrm{p}$.

Malrain F., Matterne V., Méniel P., 2002 : Les Paysans Gaulois (III siècle- 52 av. J.-C.), Paris, 236 p.

MALRAIN F., BLANCQUAERT G., 2011 : « Archaelogical interventions on linear and extensive earth-moving works: what scientific value ? A close look at the second iron age », in BLANCQUAERT G., MALrain F., StäUble H., VANMERKerKe J., p. 91-110.

MARION S., BLANCQUAERT G. (DIR.), 2000 : Les installations agricoles de l'âge du Fer en France Septentrionale, Paris, ENS (Études d'histoire et d'Archéologie, VI), p. 527.

MATTERNE V., 2002 : Agriculture et alimentation végétale durant l'âge $d u$ Fer et l'époque gallo-romaine en France septentrionale, Montagnac, éd. Monique Mergoil, 310 p.

Menez Y., 1996, Une ferme de l'Armorique gauloise : Le Boisanne à Plouër-sur-Rance (Côtes-d'Armor), Paris, DAF, 58, 272 p.

Nouvel P., Barral Ph., Deffressigne S., Riquier V., Seguier J.-M., Tikonoff N., Zehner M., ACHARd-Corompt N. (COllab.), Barthélémy D. (Collab.), Drouet C. (Collab.), Moreau C. (COllab.), RAMPONi C. (COllab.)., Videau G. (COllab.), 2009: "Rythmes de création, fonctionnement et abandon des établissements ruraux de la fin de l'âge du Fer dans l'Est de la France», in BerTRAND I., Duval A., Gomez DE Soto J., Maguer P. (DIR.), p. 109-151.

Nillesse O., 2009: " Activités, métiers, vie quotidienne dans les établissements ruraux de l'Ouest de la France à travers l'instrumentum (Hallstatt D/début du Haut Empire) ", in BERTRAND I., Duval A., Gomez de Soto J., Maguer P. (DiR.), p. 45-83.

RiQuier V., Grisard J., 2011 : « An extensive surface project at Aube Logistics Park (France): the methods and initial scientific results », in Blancquaert G., Malrain F., Stäuble H., Vanmerkerke J. (ÉD.), p. 129-143.

Sauvage L., 1996 : « Existe-t-il une ferme dans le midi de la France? », in Bayard D., Collart J.-L. (Dir.), p. 297-292.

Séjalon P., Bel V., Breuil J.-Y., Pomarèdes H., 2009 : « Définition et organisation des terroirs protohistoriques de Nîmes, Gard (de la fin du VI ${ }^{\mathrm{e}}$ au $\mathrm{I}^{\mathrm{er}}$ S. av. J.-C.) », in Bertrand I., Duval A., GomeZ DE SOto J., MAguer P. (DIR.), p. 153-18o.

Vicherd G., Baudraud M.-N., De Klijn H., 1983 : « Béligneux, Ain, une vaste ferme gauloise ", Dossier Histoire et Archéologie, 78, p. 18.

Zech-Matterne V., Bouby L., Bouchette A., Cabanis M., Derreumaux M., Durand F., Marinval Ph., Pradat B., Sellami M.-F., WIETHOLD J., 2009 : « L'agriculture du VI ${ }^{\mathrm{e}}$ au $\mathrm{I}^{\mathrm{er}}$ siècle avant J.-C. en France : état des recherches carpologiques sur les établissements ruraux ", in Bertrand I., Duval A., GOMEZ DE Soto J., MAguer P. (DIR.), p. 383-416.

\section{Moulay, un oppidum de l'ouest de la Gaule revisité}

\section{Elven Le Goff}

Inrap, UMR 6566 «Centre de recherche en Archéologie,

Archéosciences, Histoire »

Catherine Moreau

Inrap

nstallée sur les rives de La Mayenne, ancienne voie navigable et véritable arête dorsale du département, Moulay est une petite commune de 900 âmes située immédiatement au sud de la ville de Mayenne, qui rassemble quant à elle près de 15 ooo habitants. Bien qu'une fortification, connue depuis le XIX ${ }^{e}$ siècle et datée seulement dans les années 1970, signalait la présence d'un habitat à fort statut social pour l'époque laténienne, rien 